\title{
Low socioeconomic status relates to asthma and wheeze, especially in women
}

\author{
Christian Schyllert (10) ${ }^{1}$, Anne Lindberg ${ }^{2}$, Linnea Hedman ${ }^{1,3}$, \\ Caroline Stridsman ${ }^{3}$, Martin Andersson $^{1}$, Pinja Ilmarinen ${ }^{4}$, Päivi Piirilä ${ }^{5}$, \\ Steinar Krokstad ${ }^{6}$, Bo Lundbäck ${ }^{7}$, Eva Rönmark ${ }^{1}$ and Helena Backman (1) ${ }^{1,3}$
}

Affiliations: ${ }^{1}$ Dept of Public Health and Clinical Medicine, Section of Sustainable Health/the OLIN unit, Umeå University, Umeå, Sweden. ${ }^{2}$ Dept of Public Health and Clinical Medicine, Division of Medicine, Umeå University, Umeå, Sweden. ${ }^{3}$ Dept of Health Sciences, Division of Nursing, Luleå University of Technology, Luleå, Sweden. ${ }^{4}$ Dept of Respiratory Medicine, Seinäjoki Central Hospital, Seinäjoki, Finland. ${ }^{5}$ Unit of Clinical Physiology, Helsinki University Hospital and University of Helsinki, Helsinki, Finland. ${ }^{6}$ HUNT Research Centre, Dept of Public Health and Nursing, Faculty of Medicine and Health Sciences, Norwegian University of Science and Technology, Levanger, Norway. ${ }^{7}$ Krefting Research Centre, Institute of Medicine, University of Gothenburg, Gothenburg, Sweden.

Correspondence: Christian Schyllert, Dept of Public Health and Clinical Medicine, Section of Sustainable Health, The OLIN unit, Umeå University, 90187 Umeå, Sweden. E-mail: christianschyllertđagmail.com

ABSTRACT Low socioeconomic status (SES) has been associated with asthma and wheezing. Occupational group, educational level and income are commonly used indicators for SES, but no single indicator can illustrate the entire complexity of SES. The aim was to investigate how different indicators of SES associate with current asthma, allergic and nonallergic, and asthmatic wheeze.

In 2016, a random sample of the population aged 20-79 years in Northern Sweden were invited to a postal questionnaire survey, with $58 \%$ participating $(\mathrm{n}=6854)$. The survey data were linked to the national Integrated Database for Labour Market Research by Statistics Sweden for the previous calendar year, 2015. Included SES indicators were occupation, educational level and income.

Manual workers had increased risk for asthmatic wheeze, and manual workers in service for current asthma, especially allergic asthma. Primary school education associated with nonallergic asthma, whereas it tended to be inversely associated with allergic asthma. Low income was associated with asthmatic wheeze. Overall, the findings were more prominent among women, and interaction analyses between sex and income revealed that women, but not men, with low income had an increased risk both for asthmatic wheeze and current asthma, especially allergic asthma.

To summarise, the different indicators of socioeconomic status illustrated various aspects of associations between low SES and asthma and wheeze, and the most prominent associations were found among women.

@ERSpublications

Occupation, educational level and income each mirror different aspects of the association between socioeconomic status and asthma and asthmatic wheeze. This is most pronounced among women. Health-related social inequities should not be underestimated. https://bit.ly/2ByuFRz

Cite this article as: Schyllert C, Lindberg A, Hedman L, et al. Low socioeconomic status relates to asthma and wheeze, especially in women. ERJ Open Res 2020; 6: 00258-2019 [https://doi.org/ 10.1183/23120541.00258-2019].

This article has supplementary material available from openres.ersjournals.com.

Received: 19 Sept 2019 | Accepted after revision: 31 May 2020

Copyright $\odot$ ERS 2020. This article is open access and distributed under the terms of the Creative Commons Attribution Non-Commercial Licence 4.0 


\section{Introduction}

Asthma is one of the most common chronic respiratory diseases, with a prevalence of around $10 \%$ among adults in the Nordic countries [1,2]. Risk factors include allergic sensitisation, obesity and family history of asthma, but indoor and outdoor air pollution are also of importance [3-6]. Globally, low socioeconomic status (SES) and poverty are associated with asthma and respiratory symptoms $[7,8]$. Common indicators of SES are occupation, educational level and income, each of them measuring different aspects. However, no single measure can encompass the entire relationship between SES and health-related outcomes $[9,10]$.

The occupational group of manual workers had an increased risk for recurrent wheeze, a symptom common in asthma, according to a cross-sectional postal questionnaire survey conducted in Finland, Estonia and Sweden [11], and manual work in service was associated with asthma prevalence in another Swedish study [12]. Further, the European Community Respiratory Health Survey (ECRHS) found that low educational level was associated with the prevalence of asthma without atopy [8]. Similar findings have been found also in longitudinal studies, showing associations between low education and asthma [7] and more specifically asthma without atopy [8], and between manual work and asthma [12, 13]. Regarding income, birth cohort studies with national register linkage have shown associations between low family income and asthma among children in Australia [14], whereas no such association was seen in Danish children [15]. According to a systematic review published in 2015, asthma is associated with low SES, whereas allergies are associated with high SES [16]. However, whether these associations differ between men and women or between allergic and nonallergic asthma is unclear. Further, there is a lack of studies evaluating different aspects of SES such as occupation, educational level and income in the same study.

The aim was to investigate the associations between different indicators of SES and current asthma, both allergic and nonallergic, and asthmatic wheeze in a population sample, where national register data have been linked to a large postal questionnaire survey.

\section{Methods}

\section{Study population and data collection}

This was a population-based, cross-sectional study from the Obstructive Lung disease in Northern Sweden (OLIN) and the international collaboration the Nordic EpiLung studies. In 2016, a random sample of the population aged 20-79 years in Northern Sweden was invited to participate in a postal questionnaire survey. Of 12000 individuals, 11755 were found to be eligible and 6854 (58\%) of these participated. The recruitment process was similar to earlier studies within OLIN [17]. The OLIN [17, 18] and GA ${ }^{2}$ LEN (Global Allergy and Asthma European Network) [18] questionnaires were used. Data on income and educational level for the previous calendar year (2015) were collected from the Integrated Database for Labour Market Research (LISA) of Statistics Sweden [19]. Ethical approval was given by the Regional Ethical Review Board of Umeå.

\section{Definitions}

Data from the OLIN questionnaire

- Current asthma: affirmative answer to physician diagnosis of asthma and during the last 12 months at least one of the following: any wheeze, attacks of shortness of breath or use of asthma medication.

- Allergic rhinoconjunctivitis (ARC): do you have, or have you had, allergic nose or eye symptoms (hay fever)?

- Allergic asthma: current asthma with ARC.

- Nonallergic asthma: current asthma without ARC.

- Asthmatic wheeze: have you, during the last 12 months, had wheezing or whistling in your chest with shortness of breath without having a cold?

- Smoking habits: nonsmoker, ex-smoker (stopped more than 12 months ago), current smoker $<14$ or $\geqslant 15$ cigarettes per day.

- Occupational exposure to gas, dust or fumes (GDF): have you been heavily exposed to gas, dust and/or fumes at work?

- Environmental tobacco smoke (ETS) exposure: have you been heavily exposed to tobacco smoke at work or at home?

Longest held job during the professional career was used to classify socioeconomic status according to the Swedish socioeconomic classification by occupation (SEI) according to Statistics Sweden [20]: manual workers in industry (manual work industry), manual workers in service (manual work service), nonmanual employees, lower level (nonmanual lower) and intermediate level (nonmanual intermediate), 
professionals, executives and self-employed nonprofessionals (professionals), and other and unspecified (other), including students and nonclassifiable individuals.

\section{Data from the $G A^{2} L E N$ questionnaire}

- Body mass index (BMI): height/weight ${ }^{2}$ (self-reported), categorised according to the World Health Organization standard [21].

- Working status: professionally active (employed, self-employed or fulltime home person), jobless (unemployed or on sick leave), student, retired and other.

- Housing dampness: have you, at home, noticed signs of damage due to dampness?

\section{Data from the LISA database}

- Educational level: highest reached educational level according to the Swedish education nomenclature (SUN) [22], based on the International Standard of Classification of Education; compulsory school, upper secondary school, basic or advanced tertiary education.

-Income: disposable income per consumption unit (i.e. the disposable income of the household adjusted by household composition) (supplementary figure 1). Sources of annual incomes include salaries, study grants and allowances. Income was dived by quintiles (supplementary table 1).

\section{Statistics}

All analyses were carried out using IBM SPSS Statistics version 24 (IBM Corp., New York, USA). Pearson's Chi-squared test was used to compare prevalence and t-test was used to compare means between groups. Statistical significance was defined as a two-sided $\mathrm{p}<0.05$. Binomial logistic regression models were used to calculate odds ratios (ORs) and 95\% confidence interval (CI) with current asthma and asthmatic wheeze as dependent variables. Independent variables included the common possible risk factors age, smoking habits, BMI categories, housing dampness, family history of asthma, and exposure to ETS and GDF. In separate analyses, the independent variables also included SEI group, working status, educational and income levels, respectively. Separate analyses were also conducted among those professionally active and

\section{TABLE 1 Characteristics of the total study sample and among women and men}

\begin{tabular}{|c|c|c|c|c|}
\hline & Total & Women & Men & p-value ${ }^{\#}$ \\
\hline Subjects n (row \%) & $6854(100)$ & $3590(52.4)$ & $3264(47.6)$ & \\
\hline \multicolumn{5}{|l|}{ Age } \\
\hline Mean (sD) & $53.6(16.5)$ & $53.3(16.4)$ & $54.0(16.5)$ & 0.047 \\
\hline Family history of asthma & 20.9 & 24.0 & 17.4 & $<0.001$ \\
\hline \multicolumn{5}{|l|}{ BMI categories } \\
\hline Normal $(<25)$ & 42.5 & 51.0 & 33.3 & $<0.001$ \\
\hline Overweight (25-30) & 39.9 & 32.0 & 48.5 & $<0.001$ \\
\hline Obese $(>30)$ & 17.6 & 16.9 & 18.2 & 0.080 \\
\hline \multicolumn{5}{|l|}{ Smoking habits } \\
\hline Nonsmoker & 61.1 & 60.2 & 62.0 & 0.130 \\
\hline Ex-smoker & 22.9 & 22.8 & 23.0 & 0.871 \\
\hline Current smoker $<15$ cig per day & 9.6 & 10.7 & 8.5 & 0.002 \\
\hline Current smoker $\geqslant 15$ cig per day & 1.9 & 1.9 & 1.9 & 0.987 \\
\hline \multicolumn{5}{|l|}{ Environmental exposure } \\
\hline Tobacco smoke & 21.5 & 20.9 & 22.1 & 0.206 \\
\hline \multicolumn{5}{|l|}{ Occupational exposure } \\
\hline Gas, dust and/or fumes & 28.2 & 14.7 & 43.1 & $<0.001$ \\
\hline \multicolumn{5}{|l|}{ Housing standard } \\
\hline Dampness & 6.9 & 6.8 & 7.0 & 0.718 \\
\hline \multicolumn{5}{|c|}{ Asthma, asthmatic wheeze and allergic rhinoconjunctivitis } \\
\hline Current asthma & 10.5 & 11.8 & 8.9 & $<0.001$ \\
\hline Allergic asthma & 6.3 & 7.2 & 5.5 & 0.004 \\
\hline Nonallergic asthma & 4.1 & 4.7 & 3.5 & 0.013 \\
\hline Asthmatic wheeze & 7.1 & 7.6 & 6.5 & 0.067 \\
\hline Allergic rhinoconjunctivitis & 24.8 & 25.4 & 24.1 & 0.217 \\
\hline
\end{tabular}

Data are presented as column percentages, unless otherwise stated. ${ }^{\#}$ : comparing sexes by t-test for age, otherwise Pearson's Chi-squared test was used. BMI: body mass index; cig: cigarettes. 
stratified by sex. Multinomial logistic regression analyses were used to calculate OR and 95\% CI for the mutually exclusive categories of allergic and nonallergic asthma with no asthma as reference, with independent variables as described above. Unadjusted ORs are labelled OR and adjusted ORs (aOR) are labelled as such in the results section.

Relative index of inequality (RII) was calculated for educational levels and income groups by ranking the population sample from the most disadvantaged subgroup to the most advantaged (i.e. from lowest to the highest ranked category). The population of each category is considered in terms of its range in the frequency distribution, and the midpoint of this range (supplementary figure 2) [23].

Interaction analysis between equalised disposable income and sex was made with the fifth (highest) income group (supplementary table 1) as a reference category, regardless of sex. In these interaction analyses, the two lowest income categories were labelled "low income", and the third and fourth income categories were labelled "medium income". These groups were divided by sex into mutually exclusive groups: 1) men with medium income, 2) women with medium income, 3) men with low income and 4) women with low income, which were all compared to the reference category of "high income".

\section{Results}

\section{Sample characteristics}

In the study sample, $52.4 \%$ were women, $61.1 \%$ were nonsmokers and $42.5 \%$ had $\mathrm{BMI}<25$ (table 1 ). More women than men had tertiary education $(34.9 \%$ versus $22.8 \%, \mathrm{p}<0.001)$ but women had lower disposable income than men (table 2). Both current and former smoking as well as BMI $>25$ were more common among those with lower education (supplementary figure 3 ).

\section{Socioeconomic status in relation to asthma and asthmatic wheeze}

Current asthma and asthmatic wheeze

The occupational SEI group of manual work in service was, compared to professionals and executives, associated with current asthma, OR 1.52 (95\% CI 1.13-2.05) and aOR 1.41 (1.03-1.91). When adjusting also for GDF exposure and further additionally for ETS exposure the aORs were 1.36 (1.00-1.85) and 1.33

\begin{tabular}{|c|c|c|c|c|}
\hline & Total & Women & Men & p-value \\
\hline \multicolumn{5}{|l|}{ Socioeconomic classification by occupation (SEI)" } \\
\hline Professionals, executives and self-employed & 10.8 & 7.9 & 14.0 & $<0.001$ \\
\hline Nonmanual employees (intermediate) & 20.1 & 24.0 & 15.8 & $<0.001$ \\
\hline Non-manual employees (lower) & 10.2 & 13.0 & 7.1 & $<0.001$ \\
\hline Manual workers in service & 22.1 & 29.9 & 13.6 & $<0.001$ \\
\hline Manual workers in industry & 15.2 & 2.9 & 28.7 & $<0.001$ \\
\hline Students & 4.2 & 4.3 & 4.0 & 0.453 \\
\hline Other and unspecified & 17.4 & 18.0 & 16.7 & 0.176 \\
\hline \multicolumn{5}{|l|}{ Working status } \\
\hline Professionally active & 54.4 & 53.6 & 55.4 & 0.123 \\
\hline Jobless & 5.9 & 6.2 & 5.5 & 0.167 \\
\hline Student & 4.0 & 4.3 & 3.7 & 0.223 \\
\hline Retired & 31.8 & 30.8 & 32.9 & 0.059 \\
\hline Unspecified & 3.8 & 5.1 & 2.5 & $<0.001$ \\
\hline \multicolumn{5}{|l|}{ Educational level $^{+}$} \\
\hline Advanced tertiary & 19.7 & 24.1 & 14.9 & $<0.001$ \\
\hline Basic tertiary & 9.4 & 10.8 & 7.9 & $<0.001$ \\
\hline Upper secondary & 58.1 & 53.9 & 62.6 & $<0.001$ \\
\hline Compulsory & 12.8 & 11.1 & 14.6 & $<0.001$ \\
\hline \multicolumn{5}{|l|}{ Income ${ }^{+}$} \\
\hline High & 20.0 & 17.0 & 23.3 & $<0.001$ \\
\hline Medium-high & 20.0 & 19.6 & 20.5 & 0.377 \\
\hline Medium & 20.0 & 19.7 & 20.3 & 0.562 \\
\hline Medium-low & 20.0 & 21.6 & 18.3 & 0.001 \\
\hline Low & 20.0 & 22.1 & 17.7 & $<0.001$ \\
\hline
\end{tabular}


(0.98-1.81), respectively. However, there were no significant associations between working status, educational levels or income groups and current asthma (unadjusted results presented in figure 1, adjusted in table 3).

The occupational SEI groups of manual work in service and manual work in industry were associated with asthmatic wheeze both in unadjusted (figure 1) and adjusted analyses (table 3). The adjusted association between manual work in service and asthmatic wheeze (aOR 1.48, 95\% CI 1.02-2.14) was fairly similar when adding GDF exposure (1.43, 0.99-2.08) and also after further adding ETS exposure (1.36, 0.94-1.98) to the model. Corresponding adjustments for the association between manual work in industry and asthmatic wheeze yielded aORs of $1.50(1.02-2.21), 1.10(0.74-1.65)$ and $1.13(0.76-1.70)$, respectively.

- Current asthma

-. - Asthmatic wheeze

a)

SEl socioeconomic classification by occupation

Non-manual employees (intermediate)

- Non-manual employees (lower)

- Manual workers in industry

- Manual workers in service

Reference category: professionals and executives

b)

Educational level

Basic tertiary

Opper secondary

$\diamond$ Compulsory school

Reference category: advanced tertiary

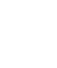

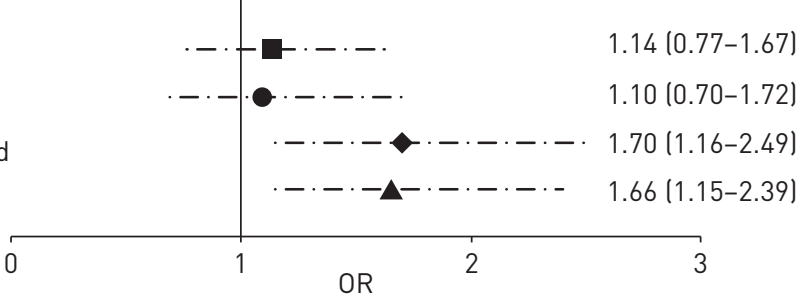

要

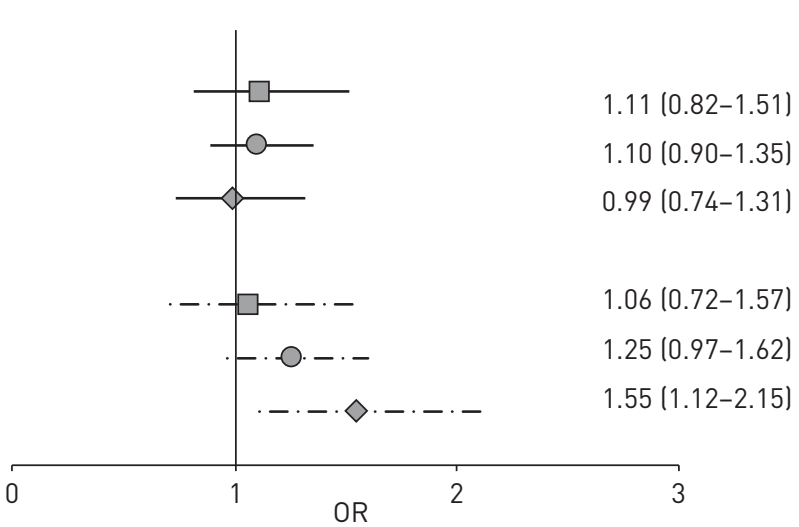

c) Income group

Medium-high

O Medium

$\diamond$ Medium-low

$\triangle$ Low

Reference category: high income

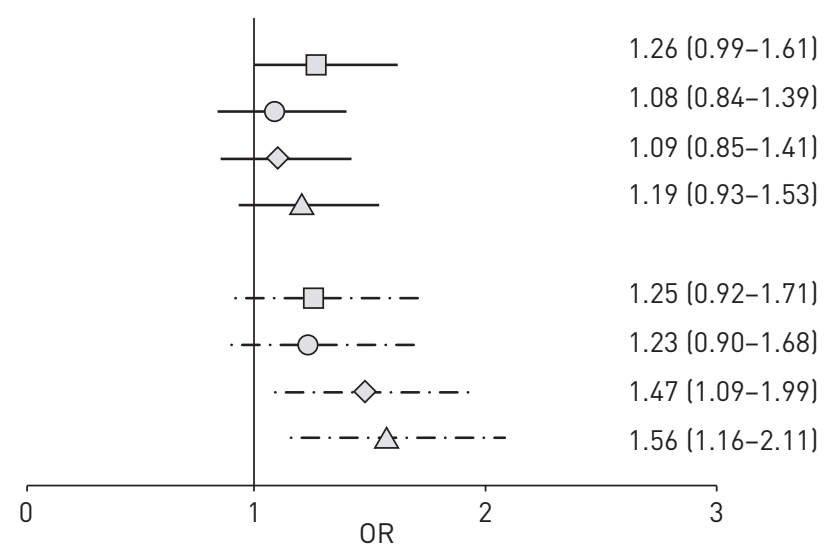

$1.24(0.91-1.69)$

$1.24(0.87-1.76)$

$1.25(0.90-1.73)$

$1.52(1.13-2.05)$

$1.14(0.77-1.67)$

$1.10(0.70-1.72)$

$1.11(0.82-1.51)$

$1.10(0.90-1.35)$

$0.99(0.74-1.31)$

$1.06(0.72-1.57)$

$1.25(0.97-1.62)$

$1.55(1.12-2.15)$

OR

FIGURE 1 Associations of a) socioeconomic classification by occupation (SEI), b) educational levels, and c) income groups, with current asthma and asthmatic wheeze. Results are presented in terms of unadjusted odds ratios with $95 \%$ confidence interval. 


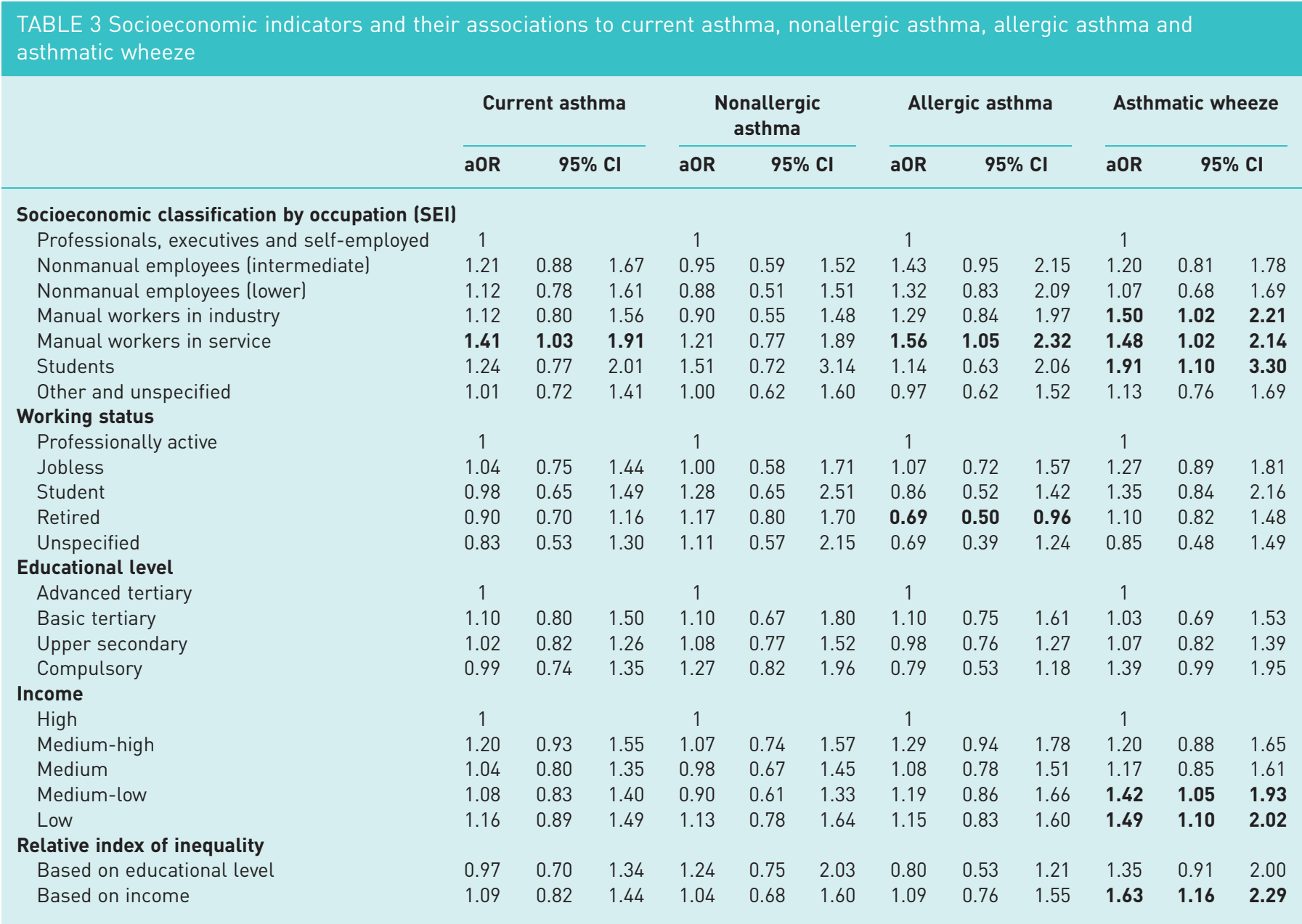

Calculated by logistic regression models and presented as aOR with $95 \% \mathrm{Cl}$, adjusted for age, smoking habits, body mass index categories, housing dampness and family history of asthma. Statistically significant results are in bold ( $p<0.05)$. aOR: adjusted odds ratio.

Besides the aforementioned occupational groups, both low education and medium-low and low income were associated with asthmatic wheeze (table 3 and figure 1). The RII was significant for asthmatic wheeze, both when based on educational level (OR 1.69, 95\% CI 1.17-2.44) and on income (OR 1.68, 95\% CI 1.21-2.33). In the adjusted analyses, RII based on income remained significant for asthmatic wheeze (table 3) and this significance persisted after further adjustment for GDF and ETS exposure.

\section{Allergic and nonallergic asthma}

When dividing current asthma into allergic and nonallergic, different patterns were revealed. The observed association between manual work in service and asthma was mainly driven by allergic asthma (OR 1.72, 95\% CI 1.17-2.54 and aOR 1.56, 1.05-2.32) (figure 2 and table 3). Also, among the professionally active, manual work in service was associated with allergic asthma (aOR 1.67, 1.05-2.65) and so was nonmanual employees (intermediate) (aOR 1.72, 1.08-2.73). Being retired was associated with lower risk for allergic asthma. Low educational level associated with increased risk for nonallergic asthma but tended to be inversely associated with allergic asthma, whereas low income was not significantly associated with either of allergic or nonallergic asthma. Medium-high income was associated with allergic asthma in the unadjusted analyses, but the significance was lost in the adjusted analyses (figure 2 and table 3). ARC showed a similar pattern to allergic asthma, but with stronger associations (data not shown). Neither the relative index for inequality based on education level nor on income were significant for current asthma, allergic or nonallergic asthma.

\section{Socioeconomic status in relation to asthma and asthmatic wheeze: patterns among} women and men

In analyses stratified by sex, no significant findings between the different indicators of SES and current asthma were found. The associations between manual work in service and allergic asthma were of similar 
- - - Nonallergic asthma

.......... Allergic asthma

OR $(95 \% \mathrm{Cl})$

a)

SEI socioeconomic classification by occupation

Non-manual employees (intermediate)

- Non-manual employees (lower)

- Manual workers in industry

- Manual workers in service

Reference category: professionals and executives

b)

Educational level

Basic tertiary

- Upper secondary

$\diamond$ Compulsory school

Reference category: advanced tertiary

c) Income group

Medium-high

O Medium

$\diamond$ Medium-low

$\triangle$ Low

Reference category: high income
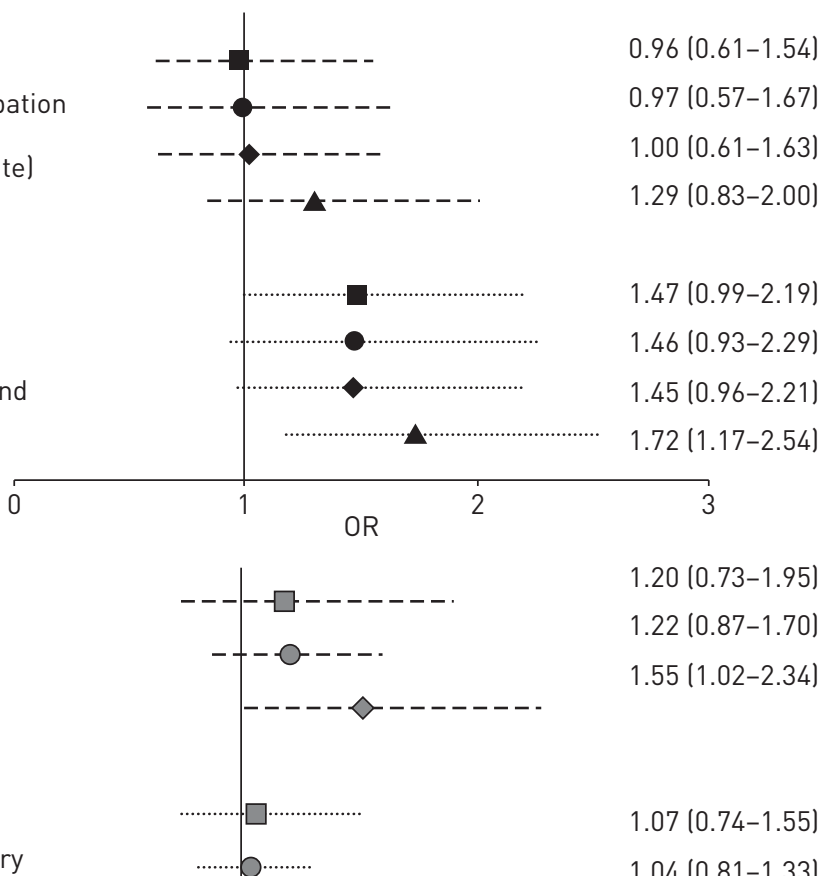

$1.20(0.73-1.95)$

$1.22(0.87-1.70)$

$1.55(1.02-2.34)$

$1.07(0.74-1.55)$

$1.04(0.81-1.33)$

$0.69(0.47-1.02)$
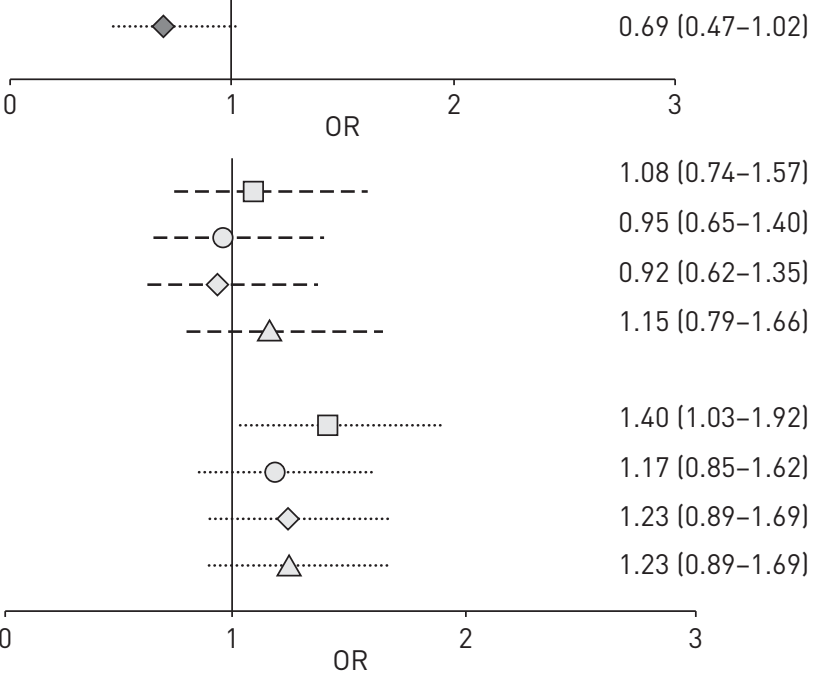

OR

FIGURE 2 Associations of a) socioeconomic classification by occupation (SEI), b) educational levels, and c) income groups, with nonallergic and allergic asthma, respectively. Results are presented in terms of unadjusted odds ratios with $95 \%$ confidence interval.

magnitude, although not significant, among women (OR 1.63, 95\% CI 0.93-2.86) and men (OR 1.56, 0.87-2.80). Regarding asthmatic wheeze, manual workers in industry had a significantly increased risk among both women (OR 2.65, 1.27-5.52) and men (OR 1.69, 1.04-2.73). The corresponding aORs for asthmatic wheeze were 2.09 (0.99-4.44) among women and 1.53 (0.94-2.48) among men (supplementary tables 2 and 3). Low income was associated with asthmatic wheeze among women but not among men, and these significant findings pertained also in the adjusted analyses (aOR 1.93, 95\% CI 1.25-2.99 among women, supplementary table 2).

Interaction analyses based on sex and income revealed that compared to those with high income, the group "low-income women" had significantly increased risk for current asthma, allergic asthma and asthmatic wheeze, whereas no such associations were found for low-income men (figure 3). These findings persisted after further adjustment for GDF and ETS exposure. 


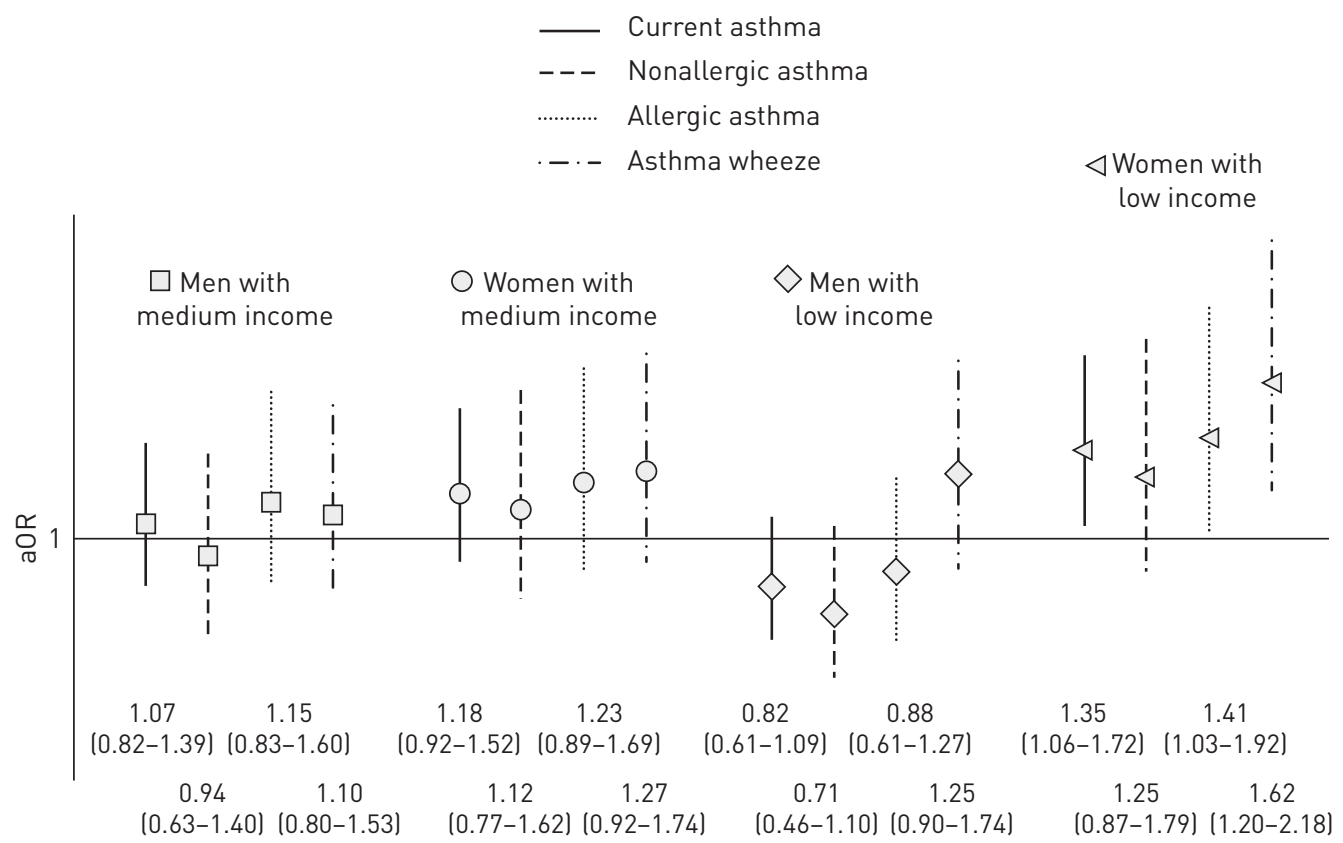

FIGURE 3 Interaction analysis between income and sex in association with current asthma, nonallergic asthma, allergic asthma, and asthmatic wheeze. Results presented in terms of adjusted odds ratios (aOR) with $95 \%$ confidence interval from logistic regression models with high income as reference category. The models are adjusted for age, smoking habits, body mass index categories, house dampness and family history of asthma.

\section{Discussion}

This population-based Swedish study demonstrates how different indicators of SES based on occupation, education and income were associated with asthma. Manual workers in both service and industry had increased risk for asthmatic wheeze, and manual workers in the service industry had increased risk for current asthma, an association that seemed to be driven by allergic asthma. Compared with advanced tertiary education, primary school education was associated with nonallergic asthma, whereas it tended to be inversely associated with allergic asthma. Individuals with low income had higher risk for asthmatic wheeze, significantly among women but not men. Women with low income had higher risk for current asthma, especially allergic asthma and asthmatic wheeze compared to those with high income, whereas no corresponding findings were found among men.

To our knowledge, there are few studies including occupation, education and income as single-measure indicators of SES in the same study, and importantly we could demonstrate some differences in their associations with asthma and wheeze. SES is certainly a diverse measure; however, it is sometimes analysed by combining different indicators such as income, education, occupation and deprivation on a country or area level into a composite measure. In a review of studies in high- and medium-income countries using a composite measure, it was concluded that low SES is more often associated with asthma, whereas a high SES is more often associated with allergies [16]. Nevertheless, it is important to acknowledge that such composite measures have weaknesses, as different socioeconomic indicators may point in different directions, as observed in our study. Correspondingly, asthma has for instance been associated with joblessness in Iran [24] and low income in the United States [25], whereas an Israeli study on pre-recruit soldiers [26] found that high SES based on residential area was associated with asthma. Also, asthma is a heterogeneous condition, which may contribute to a complex association between SES and asthma.

In the current study, manual workers in both service and industry had increased risk for asthmatic wheeze, and manual workers in service had increased risk for current asthma. Without doubt, the association between occupation and asthma is diverse, besides the fact that occupation can be used as an indicator of SES, also occupational exposure is of importance $[4,27]$. Interestingly, the ECRHS did not find significant associations between occupational groups and asthma, but it should be noted that the overall results indicated misclassification of asthma status as one likely explanation [8]. Well in line with our results, two Swedish questionnaire surveys with 10-year follow-ups found that manual workers in service and industry were at highest risk for both prevalent asthma and recurrent wheeze at baseline and for developing asthma and wheezing during follow-up [12, 13]. In the current study, adjustment for GDF 
exposure substantially weakened the associations with manual work in industry, which illustrates that occupational exposure is a factor of importance in this context. In another Swedish study on male conscripts, where occupational title of the "family head" was used as indicator for their family SES, low SES was associated with nonallergic asthma, whereas high SES was associated with allergic asthma [28]. These results correspond well to our results of an association between manual work and current asthma and asthmatic wheeze, but are, on the other hand, also contrasting as we found stronger associations between manual work and allergic asthma than with nonallergic asthma. Importantly, the prevalence of allergic asthma has increased in Sweden from 1996 to 2006 and further to 2016 [2], which in part may explain these differences.

Among adults, asthma is more common in women, and it has been discussed that this may be explained by biological differences related to sex hormones [29]. However, as discussed above, asthma can also be caused by environmental factors, and manual workers in service include occupations such as cleaners and hairdressers, who are commonly exposed to chemicals that may increase the risk for asthma [30]. It is difficult to disentangle to what extent the driving factor is female sex, the socioeconomic circumstances for women, or both [29]. We found that the associations between manual work in service and allergic asthma remained also when adjusting for sex, and the associations between manual work in service and allergic asthma was fairly similar among women and men. Further, medium-high income was associated with allergic asthma, although it was similarly common in both sexes. Based on these findings, biological factors related to female sex are unlikely to be the only explanation. Still, the observed different pattern with regard to allergic and nonallergic asthma is difficult to explain, but one might speculate that also for example selection mechanisms such as social heritage could contribute.

Low education was associated with asthmatic wheeze but not with current asthma in our study. Educational level is nevertheless an important indicator of SES, often strongly associated with risk factors for respiratory health such as occupational exposure, smoking and high BMI [3, 31, 32]. The educational level has increased in Sweden in recent decades according to Statistics Sweden, which is also reflected by our results as about 30\% had tertiary education in 2016. However, although there was no association between current asthma and educational level in the current study, allergic and nonallergic asthma had completely different patterns. Low educational level was associated with nonallergic asthma, whereas high educational level was associated with allergic asthma. These results are well in line with the previously cited review0 concluding that ARC is associate with high SES, possibly related to higher educational levels in this group [16]. There are also studies indicating a higher prevalence of allergic sensitisation (although not for cockroach) among children in families with higher SES, which would support a "true" difference in allergic status [33].

The RII provides an appropriate approximation of the relation between SES and health outcomes. The advantage of this measure is that it allows comparisons between epidemiological studies [23]. An inequality regarding the association between low educational level and asthmatic wheeze was seen also in the current study. However, this association weakened substantially after adjustment for our list of potential risk factors, which in the context of SES can be considered mediators rather than confounders, highlighting the importance of unadjusted analyses.

Also, income provides an obvious measure for SES, and there were clear associations between the "lower" categories and asthmatic wheeze, but not with current asthma. This pattern was also illustrated by the relative index for inequality based on income, with significant associations that persisted in the adjusted analyses, supporting a strong relationship between low income and symptoms common in asthma but not as clearly with the diagnosis of asthma. These results are intriguing and indicate a social gradient in the diagnosis of asthma, as suggested also in other high-income countries [34, 35].

Moreover, the association between low income and asthmatic wheeze was seen in women but not men in the stratified analyses. Further, the interaction analyses between sex and income enabled utilisation of our entire sample, and a clear association between women with low income and current asthma, especially allergic asthma and asthmatic wheeze was revealed. In contrast, men with low income tended to have less risk for current asthma, especially nonallergic asthma, compared to those with high income, whereas there was a tendency towards an increased risk for asthmatic wheeze. Thus, the social inequalities with regard to income and asthma may potentially affect both women and men in Sweden.

A cross-sectional design, such as the current study, can demonstrate associations but not provide measures of causality. However, by linking data from national registers with a large population-based postal questionnaire survey we have combined the strengths of two methods of conducting epidemiological research. By using a validated questionnaire $[17,18]$ we have a reliable source of information on physician diagnosis of asthma and respiratory symptoms, and from national register-based data of high quality, a reliable source of income and educational level [36]. Due to lack of objective measurements, ARC was 
used as a proxy for allergy, and although the majority of young adults with allergic rhinitis are sensitised, the agreement is lower among the elderly [37]. Thus, we may have misclassified allergic asthma to some extent among the elderly, and the observed decreased risk for allergic asthma among the retired may in reality be even lower.

To summarise, we found that occupational group, educational level and income are all important indicators mirroring different aspects of SES in the complex relationship with asthma and asthmatic wheeze. Low SES based on occupation and income seemed to associate more strongly with allergic asthma than with nonallergic asthma. On the other hand, low education was associated with nonallergic asthma, whereas high education rather tended to associate with allergic asthma. Further, women with low income were at particular risk for both asthma and asthmatic wheeze, a finding not seen in men with low income. The results also indicated a social gradient in the diagnosis of asthma. Our results demonstrate the need for societal preventive measures that go beyond medical care to counteract the burden of asthma and asthmatic wheeze, and also call for future studies to increase the understanding of the driving factors for the observed sex-dependent differences.

Acknowledgements: Research assistants Ms Zandra Lundgren, Mr Bo Selinder and Ms Tessa Pohjanen of the OLIN unit and Norrbotten County Council are acknowledged for computerisation of data.

Author contributions: C. Schyllert and H. Backman carried out the statistical analyses and drafted the manuscript. A. Linderg and H. Backman contributed with the design of the study and interpretation of the analyses. B. Lundbäck and E. Rönmark contributed to the design of the study. All authors contributed to important intellectual content and critical revision of the manuscript.

Conflict of interest: C. Schyllert reports grants from the Swedish Heart-Lung Foundation, the Swedish Asthma and Allergy Association, the Northern County Councils' Regional Federation, the Swedish Foundation for Health Care Science and Allergy Research, Konsul Th.C. Berghs Stiftelse and NordForsk, during the conduct of the study. A. Lindberg reports personal fees for lectures and advisory boards from Boehringer Ingelheim and AstraZeneca, and personal fees for lectures from Novartis and Active Care, outside the submitted work. L. Hedman has nothing to disclose. C. Stridsman has nothing to disclose. M. Andersson has nothing to disclose. P. Ilmarinen reports personal fees for lectures from Astra Zeneca, Novartis, Mundipharma, Orion and GlaxoSmithKline, outside the submitted work. P. Piirilä has nothing to disclose. S. Krokstad has nothing to disclose. B. Lundbäck reports a grant for health economic study of asthma from AstraZeneca, and personal fees for lecturing and advisory board meeting participation from AstraZeneca, Novartis, GSK and Sanofi, outside the submitted work. E. Rönmark reports grants from NordForsk, the Swedish Heart-Lung Foundation, the Swedish Asthma and Allergy Association, and VISARE NORR (Northern County Councils' Regional Federation), during the conduct of the study; and grants from AstraZeneca and Sanofi, and personal fees from AstraZeneca, outside the submitted work. H. Backman reports personal fees for speaking at scientific meeting about severe asthma from AstraZeneca outside the submitted work.

Support statement: Financial support from NordForsk (an organisation under the Nordic Council of Ministers funding and facilitating Nordic cooperation on research), the Swedish Heart-Lung Foundation, the Swedish Asthma and Allergy Association, VISARE NORR (Northern County Councils' Regional Federation) and a regional agreement between Umeà University and Västerbotten County Council (Avtal om Läkarutbildning och Forskning) are gratefully acknowledged. Funding information for this article has been deposited with the Crossref Funder Registry.

\section{References}

1 European Respiratory Society. Adult asthma. In: Gibson J, Loddenkemper R, Sibille Y, Lundbäck B, eds. European Lung White Book. 2nd Edn. Sheffield, European Respiratory Society, 2013; pp. 138-147.

2 Backman H, Räisänen P, Hedman L, et al. Increased prevalence of allergic asthma from 1996 to 2006 and further to 2016-results from three population surveys. Clin Exp Allergy 2017; 47: 1426-1435.

3 Rönmark E, Andersson C, Nyström L, et al. Obesity increases the risk of incident asthma among adults. Eur Respir J 2005; 25: 282-288.

4 Torén K, Blanc PD. Asthma caused by occupational exposures is common - A systematic analysis of estimates of the population-attributable fraction. BMC Pulm Med 2009; 9: 7.

5 Andersson M, Modig L, Hedman L, et al. Heavy vehicle traffic is related to wheeze among schoolchildren: a population-based study in an area with low traffic flows. Environ Health 2011; 10: 91.

6 Hulin M, Simoni M, Viegi G, et al. Respiratory health and indoor air pollutants based on quantitative exposure assessments. Eur Respir J 2012; 40: 1033-1045.

7 Eagan TML, Gulsvik A, Eide GE, et al. The effect of educational level on the incidence of asthma and respiratory symptoms. Respir Med 2004; 98: 730-736.

8 Ellison-Loschmann L, Sunyer J, Plana E, et al. Socioeconomic status, asthma and chronic bronchitis in a large community-based study. Eur Respir J 2007; 29: 897-905.

9 Galobardes B, Shaw M, Lawlor DA, et al. Indicators of socioeconomic position (part 2). J Epidemiol Community Health 2006; 60: 95-101.

10 Galobardes B, Shaw M, Lawlor DA, et al. Indicators of socioeconomic position (part 1). J Epidemiol Community Health 2006; 60: 7-12.

11 Pallasaho P, Lindström M, Pölluste J, et al. Low socio-economic status is a risk factor for respiratory symptoms: a comparison between Finland, Sweden and Estonia. Int J Tuberc Lung Dis 2004; 8: 1292-1300.

12 Ekerljung L, Sundblad BM, Ronmark E, et al. Incidence and prevalence of adult asthma is associated with low socio-economic status. Clin Respir J 2010; 4: 147-156. 
13 Hedlund U, Eriksson K, Rönmark E. Socio-economic status is related to incidence of asthma and respiratory symptoms in adults. Eur Respir J 2006; 28: 303-310.

14 Kozyrskyj AL, Kendall GE, Jacoby P, et al. Association between socioeconomic status and the development of asthma: analyses of income trajectories. Am J Public Health 2010; 100: 540-546.

15 Hammer-Helmich L, Linneberg A, Thomsen SF, et al. Association between parental socioeconomic position and prevalence of asthma, atopic eczema and hay fever in children. Scand J Public Health 2014; 42: 120-127.

16 Uphoff E, Cabieses B, Pinart M, et al. A systematic review of socioeconomic position in relation to asthma and allergic diseases. Eur Respir J 2015; 46: 364-374.

17 Backman H, Hedman L, Jansson SA, et al. Prevalence trends in respiratory symptoms and asthma in relation to smoking - two cross-sectional studies ten years apart among adults in northern Sweden. World Allergy Organ J 2014; 7: 1 .

18 Ekerljung L, Rönmark E, Lötvall J, et al. Questionnaire layout and wording influence prevalence and risk estimates of respiratory symptoms in a population cohort. Clin Respir J 2013; 7: 53-63.

19 Statistics Sweden. Longitudinell integrationsdatabas för Sjukförsäkrings- och Arbetsmarknadsstudier (LISA). Örebro, 2016.

20 Statistics Sweden. MIS 1982:4. Socioekonomisk indelning (SEI). Stockholm, 2007.

21 World Health Organization. WHO/Europe Nutrition - Body mass index - BMI. www.euro.who.int/en/healthtopics/disease-prevention/nutrition/a-healthy-lifestyle/body-mass-index-bmi\# Date last accessed: October 17, 2018 Date last updated: 2018.

22 Statistics Sweden. 2000. Svensk utbildningsnomenklatur SUN 2000.

23 Moreno-Betancur M, Latouche A, Menvielle G, et al. Relative index of inequality and slope index of inequality: a structured regression framework for estimation. Epidemiology 2015; 26: 518-527.

24 Masoompour SM, Mahdaviazad H, Ghayumi SMA. Asthma and its related socioeconomic factors: the Shiraz Adult Respiratory Disease Study 2015. Clin Respir J 2018; 12: 2110-2116.

25 Chittleborough CR, Taylor AW, Dal Grande E, et al. Gender differences in asthma prevalence: Variations with socioeconomic disadvantage. Respirology 2010; 15: 107-114.

26 Gordon B, Hassid A, Bar-Shai A, et al. Association between asthma and body mass index and socioeconomic status: a cross-sectional study on 849659 adolescents. Respirology 2016; 21: 95-101.

27 Balmes J, Becklake M, Blanc P, et al. American Thoracic Society Statement: occupational contribution to the burden of airway disease. Am J Respir Crit Care Med 2003; 167: 787-797.

28 Bråbäck L, Hjern A, Rasmussen F. Social class in asthma and allergic rhinitis: a national cohort study over three decades. Eur Respir J 2005; 26: 1064-1068.

29 Melgert BN, Ray A, Hylkema MN, et al. Are there reasons why adult asthma is more common in females? Curr Allergy Asthma Rep 2007; 7: 143-150.

30 Schyllert C, Rönmark E, Andersson M, et al. Occupational exposure to chemicals drives the increased risk of asthma and rhinitis observed for exposure to vapours, gas, dust and fumes: a cross-sectional population-based study. Occup Environ Med 2016; 73: 663-669.

31 Bilal U, Beltrán P, Fernández E, et al. Gender equality and smoking: a theory-driven approach to smoking gender differences in Spain. Tob Control 2016; 25: 295-300.

32 Wang Y, Beydoun MA. The obesity epidemic in the United States--gender, age, socioeconomic, racial/ethnic, and geographic characteristics: a systematic review and meta-regression analysis. Epidemiol Rev 2007; 29: 6-28.

33 Kim J, Hahm MI, Lee SY, et al. Sensitization to aeroallergens in Korean children: a population-based study in 2010. J Korean Med Sci 2011; 26: 1165-1172.

34 Williams RL, Romney C, Kano M, et al. Racial, gender, and socioeconomic status bias in senior medical student clinical decision-making: a national survey. J Gen Intern Med 2015; 30: 758-767.

35 Chapman EN, Kaatz A, Carnes M. Physicians and implicit bias: how doctors may unwittingly perpetuate health care disparities. J Gen Intern Med 2013; 28: 1504-1510.

36 United Nations Economic Commission for Europe. Register-based statistics in the Nordic countries: review of best practices with focus on population and social statistics. New York, Geneva, United Nations, 2007.

37 Warm K, Hedman L, Lindberg A, et al. Allergic sensitization is age-dependently associated with rhinitis, but less so with asthma. J Allergy Clin Immunol 2015; 136: 1559-1565. 